\title{
The Nuclear Physics of the Neutron Star Crust
}

\author{
Andrew W. Steiner*t \\ Joint Institute for Nuclear Astrophysics and National Superconducting Cyclotron Laboratory \\ Department of Physics and Astronomy, Michigan State University E. Lansing, MI 48824-2320 \\ E-mail: steinera@nscl.msu.edu
}

\begin{abstract}
The neutron star crust, the 1 kilometer region on the surface composed of nuclei and superfluid neutrons, is the physical location of the majority of the phenomena observed in neutron stars. The composition of the crust is a fundamental input for the evolution of isolated and accreting neutron stars, and is important for the description of rp-process nucleosynthesis, gravitational waves generated from accretion, and giant gamma-ray flares. I show that the equation of state and the composition of the crust depend critically on the description of low-density neutron matter nuclear symmetry energy. Because of this dependence, experiments measuring masses of neutronrich nuclei and experiments measuring the symmetry energy are important in determining the composition of the crust. A self-consistent model of the neutron star crust is created, which allows one to properly understand the extent to which uncertainties in the nuclear physics generate uncertainties in the composition [1].
\end{abstract}

10th Symposium on Nuclei in the Cosmos

July 27 - August 12008

Mackinac Island, Michigan, USA

\footnotetext{
* Speaker.

${ }^{\dagger}$ This work is supported by Joint Institute for Nuclear Astrophysics at MSU under NSF-PFC grant PHY 02-16783.
} 


\section{Introduction}

The inner crust of a cold neutron star can be defined as the region between the density where neutrons drip out of nuclei (about $4 \times 10^{11} \mathrm{~g} / \mathrm{cm}^{3}$ ) and the density for the transition to homogeneous nucleonic matter at about half of the nuclear saturation density. This region is sensitive to the nuclear physics input because the nature of the crust is determined by the structure of neutron-rich nuclei and the energetics of the surrounding dripped neutrons. In this work, the dependence of the description of the neutron-rich nuclei and the dripped neutrons on the equation of state (EOS) of homogenous nucleonic matter is examined.

The inner crust is of broad interest because a large variety of astrophysical observations are dependent on and sensitive to the properties of the neutron star crust. One recent motivation is the suggestion that the giant flares in Soft Gamma-Ray repeaters trigger seismic events in the neutron star crust and are sensitive to the shear modulus of the crust crust $[2,3,4]$. The shear modulus, in turn, is sensitive to the composition of the neutron star crust and the relative magnitude of the proton and neutron numbers of the nuclei in the inner crust. Neutrino and photon opacities are sensitive to the properties of the nuclei in the inner crust. For example, neutrino-nucleus scattering, which scales like $A^{2}$, is the most important neutrino process during the lepton-trapped phase of a Type II supernova (see Ref. [5] for a recent review). Finally, the cooling and evolution of neutron star crusts depends on the both the size of the crust [6] and by its transport properties [7, 8], which are both related to the composition. These astrophysical connections motivate the study of the magnitude of the uncertainty of the properties of the inner crust which come from present uncertainties in the nuclear physics inputs.

While microscopically-based models of the nuclei are of great interest because they can disentagle important effects which are not easily treated in a classical approach, a microscopic approach can also make it more difficult to understand the physical principles which guide the nature of the inner neutron star crust. Since the purpose is only to estimate the uncertainties from the nuclear physics input to the EOS, a liquid-drop model quite similar to that described in Refs. $[9,10]$ is used. More microscopic models for the crust have been developed (see the pioneering work of Ref. [11] and recent efforts in Refs. [12,13]) and it is expected that these results on the sensitivity to the EOS of homogeneous nucleonic matter will apply to some extent in these models as well.

\section{The Equations of State}

The EOS from Ref. [14] (APR) is used, which was obtained from variational chain summation calculations of the equation of state using a realistic nucleon-nucleon interaction. Also, a "typical" relativstic field-theoretical model is utilized (review in Ref. [15]), NL4 [16] which was fit to nuclei. In order to compare with the model of Ref. [17] the Skyrme [18] model SLy4 [19] is used, and in order to compare with the model from Ref. [20] the Skyrme model SkM* [21] is used.

APR is expected to be particularly good for neutron matter at low densities, because it is directly computed from an interaction which reproduces the two-body nucleon-nucleon phase shifts. The model SLy4 also has a good neutron matter EOS because it was fit to both nuclei and lowdensity neutron matter. The NL4 and $\mathrm{SkM}^{*}$ models were only fit to nuclei and low-density neutron matter are less constrained. $\mathrm{SkM}^{*}$ happens to have a neutron matter EOS which is somewhat closer 
to APR than NL4. Like the SLy4 interaction, relativistic models are also able to reproduce, at some level, the more accurate low-density neutron matter EOS found in APR and SLy4, as was demonstrated by the RAPR model in Ref. [22] and the FSUGold model [23, 24].

\section{The Crusts}

In order to determine the composition and properties of the crust, the energy at a fixed density as a function of the proton number and atomic number of nuclei, and the number density of dripped neutrons, $n_{n, \text { drip }}$, is minimized. The energy of matter in the neutron star crust is given by

$$
\begin{aligned}
\varepsilon\left(Z, A, n_{n, \text { drip }}\right)= & \left(n_{n}+n_{p}\right) \chi B(Z, A) / A+ \\
& (1-\chi) \varepsilon_{\text {drip }}\left(n_{n, \text { drip }}\right)+\varepsilon_{\mathrm{el}}\left(n_{e}\right)
\end{aligned}
$$

where $B(Z, A)$ is the binding energy of a nucleus with proton number $Z$ and mass number $A$, $\varepsilon_{\text {drip }}\left(n_{n \text {,drip }}\right)$ is the energy density of neutron matter evaluated at the local dripped neutron number density, and $\varepsilon_{\mathrm{el}}\left(n_{e}\right)$ is the electron energy density evaluated at the electron number density. The total energy is minimized over the three parameters $Z, A$, and $n_{n \text {,drip }}$ at each density. The volume fraction of matter inside nuclei, $\chi$, is determined from the relation

$$
n_{B}=\chi\left(n_{n}+n_{p}\right)+n_{n, \text { drip }}(1-\chi)
$$

It is useful to examine the crust in terms of a symmetry energy with the form $E_{\mathrm{sym}}=A_{\mathrm{sym}}\left(n / n_{0}\right)^{2 / 3}+$ $B_{\text {sym }}\left(n / n_{0}\right)^{\gamma}$. For an effective mass of about $0.7 M, A_{\text {sym }}$ is about $17 \mathrm{MeV}$, and then $B_{\text {sym }}$ and $\gamma$ are parameters which dictate the magnitude of the symmetry energy at the saturation density and the density dependence of the symmetry energy, respectively.

To compare the effect of the uncertainty in the symmetry energy, Fig. 1 shows the composition for the neutron star crust as a function of density for the schematic equations of state with different symmetry energies. The naive expectation is that a stronger symmetry energy tends to encourage nuclei to become more isospin-symmetric. This is coupled, however, with the fact that an increased symmetry energy will also raise the energy cost for the dripped neutrons. These two effects together could force larger, more symmetric nuclei, but this also affects the Coulomb and surface energy contributions. The variation of the composition with the value of the symmetry energy is not so clear, as the baseline model predicts larger nuclei than either models with smaller or larger values of the symmetry energy.

In order to disentangle this result, more detailed results for schematic models with different symmetry energies are given in Fig. 1 at a fixed density of $n_{B}=0.01 \mathrm{fm}^{-3}$. Beginning with the larger symmetry energy (with a value at saturation of $34 \mathrm{MeV}$ ) and proceeding downward, the expected result is obtained: lower symmetry energies allow the system to create more isospinasymmetric nuclei. At low enough symmetry energies, however, this becomes too costly as the electron contribution to the energy increases (the proton number decreases, but the volume fraction occupied by nuclei increases, thus the electron density must increase). Instead, the system reponds by moving neutrons out of the nuclei, which lowers the electron contribution, even though it increases the contributions from nuclei and the dripped neutrons. This is allowed, in part, because the nuclei are able to maintain a relatively constant energy. They can do this because the surface 

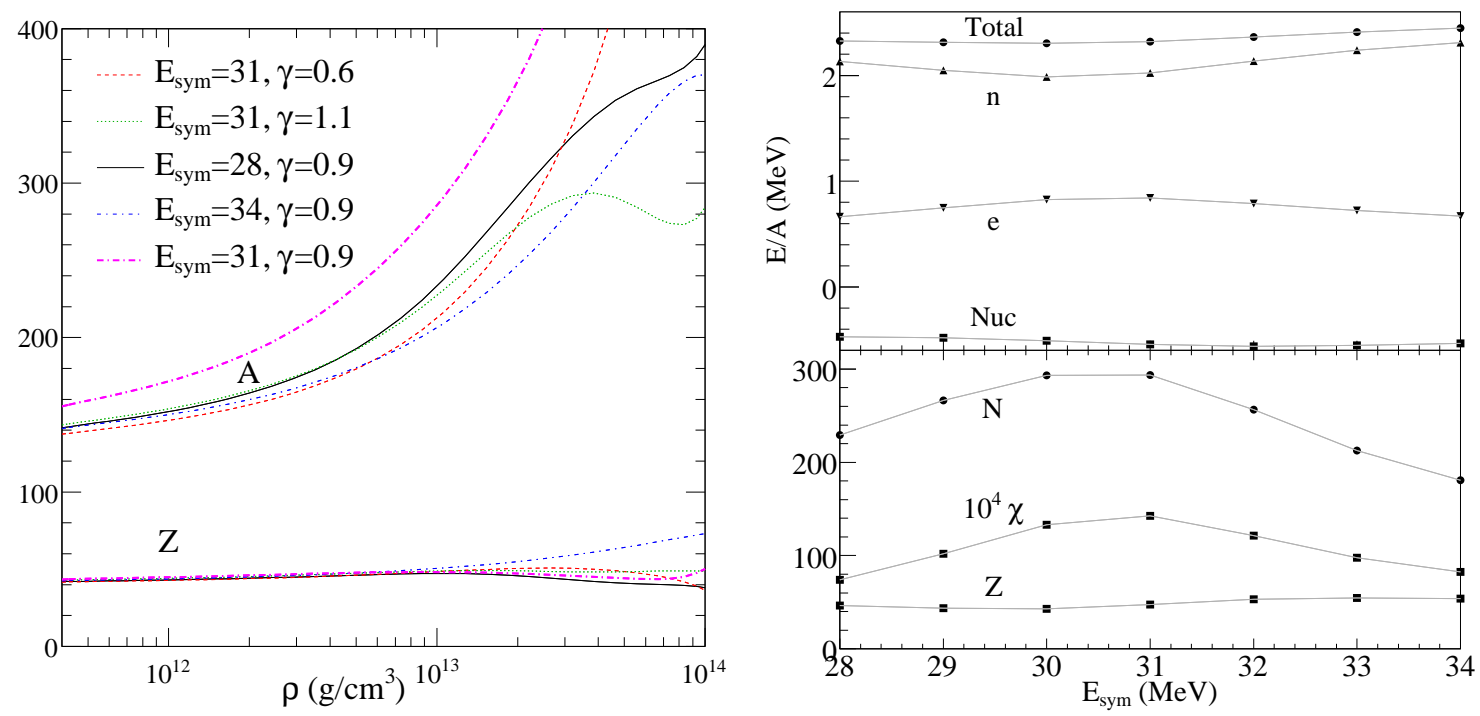

Figure 1: A comparison of the composition of the crust given different symmetry energies. The bold solid line is the baseline model, the dashed and dotted lines show the variation in $\gamma$, and the dashed-dotted and thin solid line give the variation in the magnitude of the symmetry energy at the saturation density. A comparison of the composition of the crust given different values of the symmetry energy at the saturation density at a fixed density of $n_{B}=0.01 \mathrm{fm}^{-3}$. The top panel gives the total binding energy per baryon, and the separate contributions from dripped neutrons ("n"), electrons ("e"), and nuclei ("Nuc"). The bottom panel shows the neutron and proton number of nuclei as well as the volume fraction, $\chi$.

and Coulomb energy cost is cancelled by the bulk energy gain which results from making nuclei with a larger (in absolute magnitude) bulk binding energy.

\section{Conclusions}

The composition of the neutron star crust is still partially unknown, due to uncertainties in the nuclear mass formula and the equation of state. The composition (and to a lesser extent, the overall pressure) is quite sensitive to the equation of state of low-density neutron matter, and the nuclear symmetry energy, both its magnitude and its density dependence. The dependence of the composition on the symmetry energy is not monotonic, as models with moderate symmetry energies can have larger nuclei than models with lower or higher symmetry energies. To the extent to which neutron stars depend on the composition, this means that it is important to explore the full range of variation in the crust allowed by the present knowledge of the input nuclear physics, while ensuring that the EOS is constrained by what is already known about the EOS of low-density neutron matter. Nuclear experiments will continue to provide better constraints on the symmetry energy, including from the PREX experiment $[25,26]$ to measure the neutron skin thickness of lead at Jefferson Lab and from intermediate-energy heavy-ion collisions as has been done in Ref. [27].

It remains to be seen if these results persist in the more microscopic models which include pairing, corrections beyond the Wigner-Seitz approximation, long-range correlations, and better treatments of the nuclear structure. Also important is the effect of the symmetry energy on the nuclear pasta [29], described elsewhere in these proceedings. 


\section{References}

[1] A. W. Steiner, Phys. Rev. C 77 (2008) 035805.

[2] C. Thompson and R. C. Duncan, Mon. Not. Roy. Astron. Soc. 275 (1995) 255.

[3] T. E. Strohmayer and A. L. Watts, Astrophys. J. 653 (2006) 593.

[4] L. Samuelsson and N. Andersson, Mon. Not. Roy. Astron. Soc. 374 (2007) 256.

[5] A. Burrows, S. Reddy, and T. A. Thompson, Nucl. Phys. A 777 (2006) 356.

[6] J. M. Lattimer, K. A. van Riper, M. Prakash, and M. Prakash, Astrophys. J. 425 (1994) 802.

[7] A. Y. Potekhin, G. Chabrier, and D. G. Yakovlev, Astron. Astrophys. 323 (1997) 415 .

[8] E. F. Brown, Astrophys. J. 531 (2000) 988 .

[9] G. Baym, H. A. Bethe, and C. J. Pethick, Nucl. Phys. A 175 (1971) 225 .

[10] J. M. Lattimer, C. J. Pethick, D. G. Ravenhall, and D. Q. Lamb, Nucl. Phys. A 432 (1985) 646.

[11] J. W. Negele and D. Vautherin, Nucl. Phys. A 207 (1983) 298 .

[12] M. Baldo, E. E. Saperstein, and S. V. Tolokonnikov, Phys. Rev. C 76 (2007) 025803.

[13] W. G. Newton, J. R. Stone, and A. Mezzacappa, Journal of Physics: Conference Series 46 (2006) 408 .

[14] A. Akmal, V. R. Pandharipande, and D. G. Ravenhall, Phys. Rev. C 58 (1998) 1804.

[15] B. D. Serot and J. D. Walecka, in Adv. Nucl. Phys. (Plenum, 1989) edited by J. W. Negele and E. Vogt , vol. 16, p. 1.

[16] B. Nerlo-Pomorska and J. Sykut, Int. J. Mod. Phys. E 13 (2004) 75.

[17] F. Douchin and P. Haensel, Astron. and Astrophys. 380 (2001) 151.

[18] T. H. R. Skyrme, Nucl. Phys. 9 (1959) 615.

[19] E. Chabanat (1995) thesis (unpublished) .

[20] J. M. Lattimer, Tables for model SkM* obtained from www. astro.sunysb.edu/lattimer/EOS/main.html, (These are updates of the previous work in [28]).

[21] J. Bartel, P. Quentin, M. Brack, C. Guet, and H.-B. Høakansson, Nucl. Phys. A 386 (1982) 79.

[22] A. W. Steiner, M. Prakash, J. M. Lattimer, and P. J. Ellis, Phys. Rep. 411 (2005) 325 .

[23] B. G. Todd-Rutel and J. Piekarewicz, Phys. Rev. Lett. 95 (2005) 122501.

[24] J. Piekarewicz (2007) arXiv:0709.2699.

[25] R. Michaels, P. A. Souder, and G. M. Urciuoli (2000) Jefferson Laboratory Proposal PR-00-003 .

[26] C. J. Horowitz, S. J. Pollock, P. A. Souder, and R. Michaels, Phys. Rev. C 63 (2001) 025501.

[27] M. B. Tsang, T. X. Liu, L. Shi, P. Danielewicz, C. K. Gelbke, X. D. Liu, W. G. Lynch, W. P. Tan, G. Verde, A. Wagner, et al., Phys. Rev. Lett. 92 (2004) 062701.

[28] J. M. Lattimer and F. D. Swesty, Nucl. Phys. A 535 (1991) 331 .

[29] K. Oyamatsu and K. Iida, Phys. Rev. C 75 (2007) 015801. 